\title{
Granulomatous Lesions in Bone Marrow: A Case Series
}

\author{
Dr Akanksha Salkar ${ }^{1}$, Dr.Srikant Pankanti ${ }^{2}$, Dr. Ranjana Srikant Pankanti ${ }^{3}$ \\ Dr.Vijay Praveen Pedakontala 4 \\ ${ }^{1}$ Senior Resident, Department Of Pathology Care Hospitals Banjara Hills, Hyderabad \\ ${ }^{2}$ Senior Consultant And Head Of Pathology Department, Care Hospitals Banjara Hills, Hyderabad \\ ${ }^{3}$ Senior Consultant Pathologist, Care Hospitals Banjara Hills, Hyderabad \\ ${ }^{4}$ Senior Resident, Department of Pathology, Care Hospital, Banjara Hills, Hyderabad.
}

\begin{abstract}
Granulomas in bone marrow are an infrequent finding related to diverse diseases. We reviewed bone marrow studies over a period of 5 years, confirming the presence of granulomas in bone marrows of 17 patients. Mean age of the patients was 38 years. Male:Female ratio was 2:1. Fever was the most common presenting complaint along with, unexplained weight loss and anorexia. CBP and peripheral smear evaluation was done and showed most common finding as pancytopenia in 10 cases, bicytopenia (anemia and thrombocytopenia) in 4 and anemia in 3cases. All the patients then underwent bone marrow aspiration and biopsy procedure. Out of the 17 cases, 7 cases were diagnosed as Tuberculosis, 5 diagnosed as Sarcoidosis and 5 patients were positive for human immunodeficiency virus (HIV) infection out of which 2 were HIV-TB coinfection. Out of all 17 cases only 1 case showed epitheloid granulomas in bone marrow aspirates as well as biopsy, others showed granulomas only on biopsies. Because of the non-specificity of the morphological data, the diagnostic significance of the finding is limited, but it does serve to narrow the field of etiological possibilities.
\end{abstract}

Purpose: Granulomas in bone marrow are an infrequent finding related to various disorders. Many diseases have been implicated in the formation of granulomas in the bone marrow, but they are not specific and do not usually show characteristic features that typify a specific diagnosis.

The aim of this study was to review our clinical experience with granulomatous bone marrow diseases. We report here case series of 17 cases with bone marrow granulomas across a period of 5 years.

Study Design: This is a retrospective study which was conducted in Department of Laboratory Medicine, Pathology Section in Care Hospital Banjara Hills, Hyderabad. Clinical and laboratory data on patients with granulomas based on bone marrow aspirates and biopsy analysed in the Care hospital from year 2011 to 2016 were considered in the study.

\section{Introduction}

Bone marrow examination is an important diagnostic tool to evaluate various disorders including both neoplastic and non neoplastic hematological diseases. The bone marrow evaluation may either confirm clinically suspected disease or may provide the previously unsuspected diagnosis. The examination of bone marrow is helpful in diagnosis of hematological malignancies, granulomatous infections, Kala-azar, Human immunodeficiency virus infection ( HIV), other granulomatous disorders and hemolytic states. It is imperative for the diagnosis of pyrexia of unknown origin (PUO). Granulomas in bone marrow are an infrequent finding related to various disorders, but they are not specific and do not usually show characteristic features that typify a specific diagnosis.

\section{Material And Methods}

Clinical and laboratory data on patients with granulomas based on bone marrow aspirates and biopsy analysed in the Care hospitals, Banjara hills, Hyderabad from year 2011 to 2016 were considered in the study. Clinical history, Complete Blood Picture (CBP), ESR, blood cultures were studied and Bone marrow aspirates and biopsy were evaluated for all the cases. Bone marrow aspirate and biopsy procedure was done under complete aseptic precautions and sample collected from post superior iliac spine under local anaesthesia using trephine biopsy needle. Fever being the most common complaint, all the patients were evaluated for pyrexia of unknown origin, which consisted of a detailed clinical history and thorough physical examination. Routine laboratory investigations such as complete Hemogram, Urine examination and blood culture, Peripheral Blood smear for Malarial parasite, Liver function tests, Serum Urea \& creatinine, Widal test, Chest X-ray were performed before labeling these patients as PUO cases. Peripheral smears were stained with Leishmans stain and Bone marrow aspirates were stained with May Grunwald Giemsa (MGG) stain. For Bone marrow biopsy Hematoxilin and Eosin stain was used. Iron stores were estimated using Perls stain on aspirate smears. Ziehl- 
Neelsen stain was done on bone marrow aspirates and biopsy for all the cases. Fungal stains such as Periodic acid Schiff (PAS), Gomori Methanamine Silver (GMS) was used on bone marrow biopsy to see for any fungal organisms causing granulomas specially in HIV positive patients.

Anti nuclear antibody (ANA) test was done in all cases to rule out connective tissue disorders as the cause of granulomas. Angiotensin converting enzyme test on blood was done in cases who were AFB stain negative and Tuberculosis culture negative but had non caseating granulomas in bone marrow.

\section{Results and Observations}

We identified 17 cases having granulomas in bone marrow biopsy among 1500 bone marrow biopsies, representing $1.13 \%$ out of all the cases in our centre. Male:Female ratio was 2:1. Among the 17 patients the most common presentation was that of fever which was later found out to be pyrexia of unknown origin (PUO) fulfilling all the diagnostic criteria. Complete blood picture (CBP) showed most common finding of pancytopenia in 10 cases, 4 showed bicytopenia and 3 were anemic. 5 cases were HIV positive. They were associated with ill-defined granulomas usually in the paracortical region on bone marrow biopsy (FIG1). Out of these 5 HIV positive cases 2 were also positive for acid fast bacilli even though paucibacillary, on Ziehl Neelsen (ZN) staining of bone marrow biopsy. Out of 7 cases of tuberculosis, 3 had well defined epitheloid granulomas in paratrabecular location with few langhans giant cells and histiocytes in biopsy. 1 case showed necrotic granulomatous lesions (FIG3a \&3b) with acid fast bacilli on Ziehl- Neelson stain positive on aspirate and biopsy specimens (FIG4\&5) and TB culture positive. 3 cases had ill defined granulomas but were $\mathrm{ZN}$ stain negative on bone marrow biopsy. These 3 were however TB culture positive. These three cases had multiple mediastinal and cervical lymph node enlargement which on FNAC showed granulomatous inflammation with acid fast bacilli positive on ZN stain. 5 cases were diagnosed as Sarcoidosis on the basis of non-caseating granulomas on bone marrow biopsy (FIG 2) and high Angiotensin converting enzyme (ACE) levels and these were negative for acid fast bacilli on ZN stain and TB culture negative. They had other systemic signs of Sarcoidosis such as breathlessness, cough and showed pulmonary fibrosis on chest X- Ray and HRCT chest. Anemia and thrombocytopenia was present in all 5 cases. Other connective tissue disorders could not be associated because the cases were ANA negative. Other rare causes of granulomas in bone marrow such as malignancies, brucellosis, histoplasmosis or other connective tissue disorders, etc. could not be established in our study.

\section{Discussion}

Granulomatous diseases commonly involve multiple systems including the lungs, lymph node, liver and spleen. The finding of granulomas in the bone marrow, however, is uncommon with a reported incidence between $0.3 \%$ and $2.2 \%$ in the five published series related to this topic ${ }^{1}$. This nonspecific finding is indicative of a systemic process with an extensive list of possible causes including viral, bacterial and fungal infections; malignant diseases; autoimmune diseases; drugs and sarcoidosis. The vast majority of cases of bone marrow granulomas are attributed to infectious sources and hematologic malignancies.

In our study we also found that maximum number of cases associated with granulomas in the bone marrow were infectious (HIV \& TB) followed by Sarcoidosis.

Beckers D et al in their study stated that Epithelioid granulomas are found in $<1 \%$ of BM samples ${ }^{2}$. They are considered significant as they are associated with various infectious and neoplastic disorders. After clinicopathological and molecular work-up, a specific etiology of BM epithelioid granulomas can be attributed in up to $80 \%$ of cases. Such granulomas consist of loose particularly in severely immunocompromised patients to cohesive clusters of epithelioid histiocytes with accompanying lymphocytes, eosinophilic and neutrophilic granulocytes, and giant cells $\mathrm{s}^{3}$. In patients with infectious diseases, these granulomas mostly contain organisms, which should be actively sought for and if possible visualized (e.g. mycobacteria, histoplasmata, Bartonella henselae, treponema, Leishmania spp., toxoplasma) using special stains such as PAS, Ziehl-Neelsen, FiteForacco, Grocott, May-Grünwald-Giemsa, Warthin-Starry, etc. Immunohistochemistry or molecular genetic methods might be necessary for etiological assessment. ${ }^{4}$

Hugo L et al in 2013 and D.Kejriwal et al in 2001 also found infections to be the most common cause followed by neoplasms and connective tissue diseases for granulomas in bone marrow ${ }^{2,5}$.In our study also we got maximum cases associated with infections followed by Sarcoidosis.

D. Kejriwal et al stated that in $14 \%$ of their cases the cause of fever could not be established ${ }^{5}$. Karstaedt et al in 2001 and Sonnenberg et al in 2005 stated in their studies that Mycobacterial tuberculosis and atypical mycobacterial infections are very common in individuals who are immunocompromised as a result of HIV infection. ${ }^{6,7}$

Out of the 5 HIV positive cases in our study 2 were HIV-TB Coinfection. Both had ZN Stain positive acid fast bacilli on biopsy specimen. 
Tuberculosis (TB) is one of the main causes of morbidity and mortality in different countries. In the year 2008 an estimated 8.9-9.9 million new cases of TB and 1.1-1.7 millions deaths were estimated globally. ${ }^{8}$ According to WHO report of 2009 , an estimated $73 \%$ of new TB patients were co-infected with HIV. Approximately one-third of the 38.6 million HIVpositive individuals in the world are infected with TB and are at increased risk of developing tuberculosis ${ }^{9}$. All persons who are diagnosed with HIV infection should be tested for TB and people living with HIV and at risk for TB exposure should also be tested annually for latent TB infection. Tuberculosis is the most common cause of mortality among patients with AIDS in the world, killing approximately one of every three patients ${ }^{10}$.

The classic systemic symptoms of HIV-TB coinfection include fever, night sweats, anorexia, weight loss and weakness, however these symptoms are non-specific, sometimes resulting in delayed diagnosis or even misdiagnosis. ${ }^{11}$ Our study also shows fever, weight loss, anorexia to be the predominant symptoms.

The blood or bone marrow aspirate culture methods are regarded as a standard for the diagnosis of mycobacterial infection. Blood and bone marrow aspirates have been reported to have similar mycobacterial yields ${ }^{12}$. Sputum cultures are traditionally carried out in resource-poor countries. The sputum culture is much more sensitive than routine sputum microscopy as it is able to detect 10-100 AFB per $\mu 1 .{ }^{12}$

Bone marrow aspirates turn out to yield a comparatively high rate of TB-positive results in a setting with a high incidence of HIV-disease and TB. ${ }^{13}$

The main disadvantage of culture methods is not the need for more sophisticated resources and more qualified technicians or higher cost, but its slowness. This is certainly so with the classical egg- or agar-based media (Löwenstein-Jensen, Ogawa, Middlebrook), for which a positive result requires 3-6 weeks, limiting culture methods as the first-line diagnostic tool ${ }^{14}$. The delays in the diagnosis lead to delays in treatment initiation; a greater number of patients may potentially become infected through contact with an infected person ${ }^{16}$. The diagnostic delay of culture, together with the presence of advanced disease in many cases at presentation and the high index of suspicion among clinicians in countries with a high prevalence of $\mathrm{TB}$, often obliges the clinicians to commence anti-mycobacterial therapy based solely on clinical suspicion.

That's why bone marrow aspirate and biopsy can be considered as a quick and easy way to come to the diagnosis of granulomatous lesions. Zn Stain though not very specific but can be helpful in marking the presence of acid fast bacilli.

Sarcoidosis is an inflammatory disorder characterized by the presence of non-caseating granulomas in affected organs ${ }^{17}$. The etiology is unclear, but the presence of CD4-positive T lymphocytes and macrophages in affected organs suggests an ongoing immune response. The clinical course of sarcoidosis is highly variable. One organ may be involved or systemic disease may be present. Bone marrow involvement is rare, and isolated extrapulmonary sarcoidosis occurs in less than $5 \%$ of cases. ${ }^{18,19}[2, \underline{3}]$.

In our study also, there were non-caseating granulomas in the bone marrow biopsy alongwith anemia and thrombocytopenia. All the patients responded to systemic corticosteroids along with adalimumab in 2 patients.

\section{Conclusion}

Bone marrow aspiration and biopsy are very helpful in diagnosis of granulomatous lesions in the bone marrow which can be elemental to diagnose cases of PUO or unknown cause of Pancytopenia or anemia in immunocompetent patients and rule out HIV- TB co-infection in immunocompromised individuals.

The now recommended Xpert MTB/ RIF-test may be quite useful for rapid detection of TB in bone Marrow aspirates. However,it cannot replace the need for TB culture and costs of these advanced tests are very high.

Ethical Requirements- No animals were included in the study. Informed consent was taken from all the human participants of the study regarding invasive procedure and inclusion in research activity.

\section{Bibliography}

[1]. Eid A, Carion W, Nystrom JS. Differential Diagnoses of Bone Marrow Granuloma. West J Med 1996;164:510-515.

[2]. Brackers de Hugo L, Ffrench M, Broussolle C, et al. Eur J Intern Med. 2013; 24(5): 468-473.

[3]. Dr. Menter T and Dr. Tzankov A. Granulomatous infections in the bone marrow. Clinical Laboratory International. 2015 september.

[4]. Blanco P, Viallard JF, Parrens M, et al. Bone Marrow Fibrin Ring Granuloma.Lancet 2003; $362: 1224$.

[5]. Kejariwal D, Sarkar N, Chakraborti SK, Agarwal V, Roy S. Pyrexia of unknown origin: a prospective study of 100 cases. J Postgrad Med. 2001 Apr-Jun;47(2):104-7.

[6]. Karstaedt A, Pantanowitz L, Omar T, Sonnendecke H and Patel M. The utility of bone marrow examination in HIV-infected adults in South Africa. Q J Med.2001, 94: 101-105.

[7]. Sonnenberg P, Glynn JR, Fielding K, Murray J, Godfrey-Faussett P, Shearer S. How soon after infection with HIV does the risk of tuberculosis start to increase? A retrospective cohort study in South African gold miners. J Infect Dis. 2005 Jan 15;191(2):150-8.

[8]. World Health Organization. Global Tuberculosis Control: short update to the 2009 report. World HealthOrganization;2009. 
[9]. Oluwagbemiga AE. HIV/AIDS and family support systems: A situation analysis of people living with HIV/AIDS in Lagos State. SAHARA J 2007; 4(3): 668- 677.

[10]. Raviglione MD, Snider DE Jr, Kochi A. Global epidemiology of tuberculosis: Morbidity and mortality of a worldwide epidemic. JAMA 1995; 273(3): 220- 226

[11]. Keshinro B and Diul M . HIV-TB: epidemiology, clinical features and diagnosis of smear-negative TB. Tropical Doctor.2006,36: 68-71.

[12]. Marques M, Waites K, Jaye D, Kilby J and Reddy V . Histological examination of bone marrow core biopsy specimens has limited value in thediagnosis of mycobacterial and fungal infections in patients with the acquired immunodeficiency syndrome. Annals of Diagnostic Pathology.2000, 4: 1-6.

[13]. Akpek G, M. Lee Shing, Gagnon D, Cooley T, and Daniel G. Bone Marrow Aspiration, Biopsy, and Culture in the Evaluation of HIV-Infected Patients for Invasive Mycobacteria and Histoplasma Infections. American Journal of Hematology.2001, 67:100-106 .

[14]. Siddiqi K, Lambert $M$ and Walley J. Clinical diagnosis of smear-negative pulmonary tuberculosis in low-income countries: the current evidence. Lancet Infectious Disease.2003, 3: 288-296.

[15]. Cobbert E, Marston B, Churchyard G and De Cock K . Tuberculosis in sub- Saharan Africa: opportunities, challenges, and change in the era of antiretroviral treatment. Lancet.2006, 367: 926-937.

[16]. .Gazzola L, Zanini F, Zerbi P, Franzetti F and Gori A. The usefulness of PCR assay in diagnosing disseminated mycobacterial infection in AIDS patients. European Journal of Microbiology \& Infectious Disease.2008, 27: 163-166.

[17]. Newman LS, Rose CS, Maier LA: Sarcoidosis. N Engl J Med. 1997, 336: 1224-1234.

[18]. Eid A, Carion W, Nystrom JS: Differential diagnoses of bone marrow granuloma. West J Med. 1996, 164: 510-515.

[19]. Baughman RP, Teirstein AS, Judson MA, Rossman MD, Yeager H, Bresnitz EA, DePalo L, Hunninghake G, Jannuzzi MC, Johns CJ, McLennan G, Moller DR, Newman LS, Rabin DL, Rose C, Rybicki B, Weinberger SE, Terrin ML, Knatterud GL, Cherniak R, Case Control Etiologic Study of Sarcoidosis (ACCESS) research group: Clinical characteristics of patients in a case control study of sarcoidosis. Am J Respir Crit Care. 2001, 164: 1885-1889.

\section{Legends To Figures}

FIGURE1- Photomicrograph of Bone marrow biopsy showing epitheloid cell granuloma.( H\&E, X400)

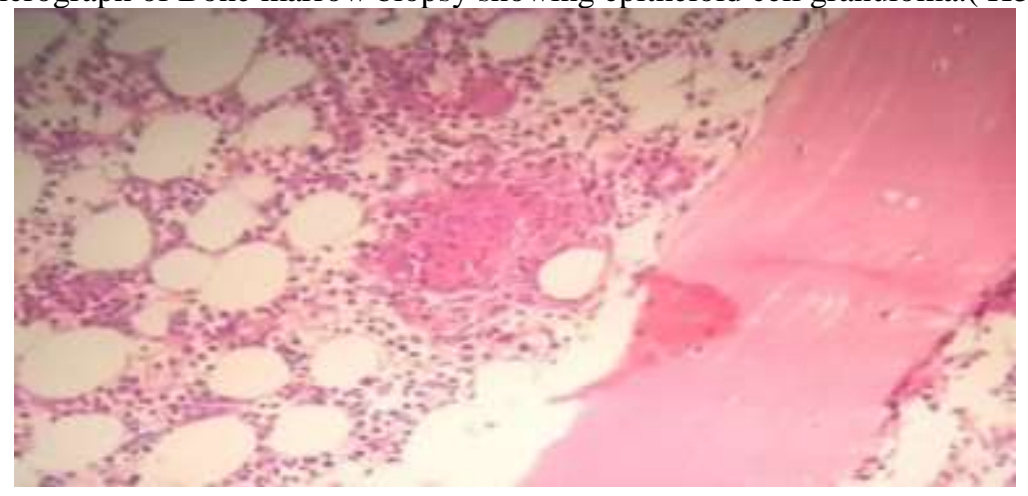

Figure 2- Photomicrograph of Bone marrow biopsy showing a non caseating granuloma composed of epitheloid cells, lymphocytes and plasma cells. (H\& E, X400)

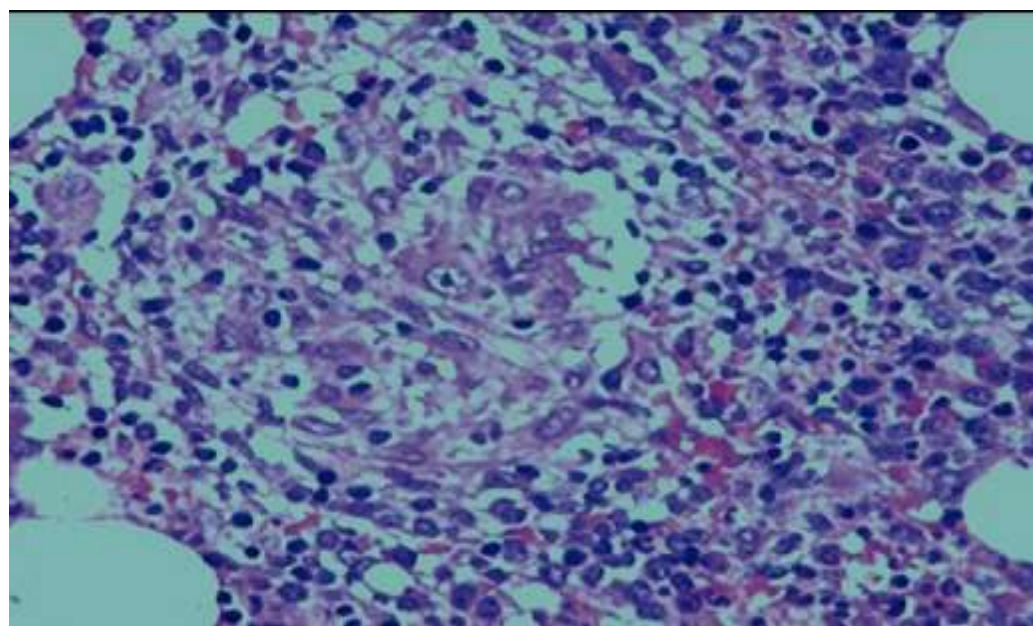

Figure 3a- Photomicrograph of bone marrow biopsy showing granuloma composed of lymphocytes, epitheloid cells and necrosis. (H \& E, X400)

Figure 3b - Photomicrograph showing necrosis. (H \& E, X1000) 

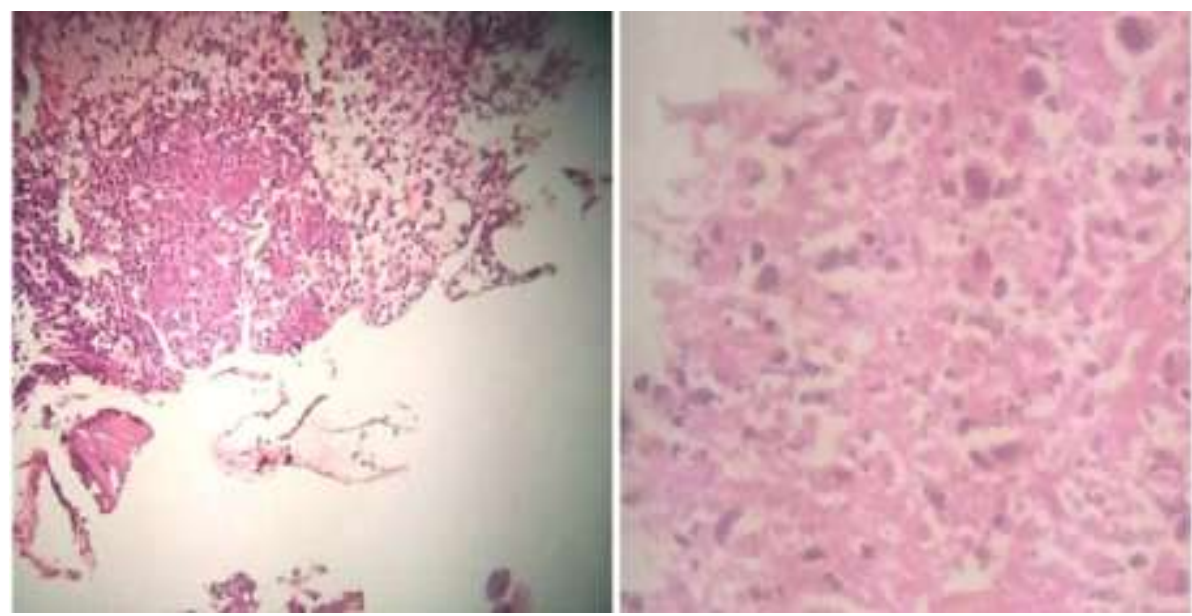

Figure 4- Photomicrograph showing many acid fast bacilli in bone marrow biopsy. (Ziehl Neelson stain, x400)

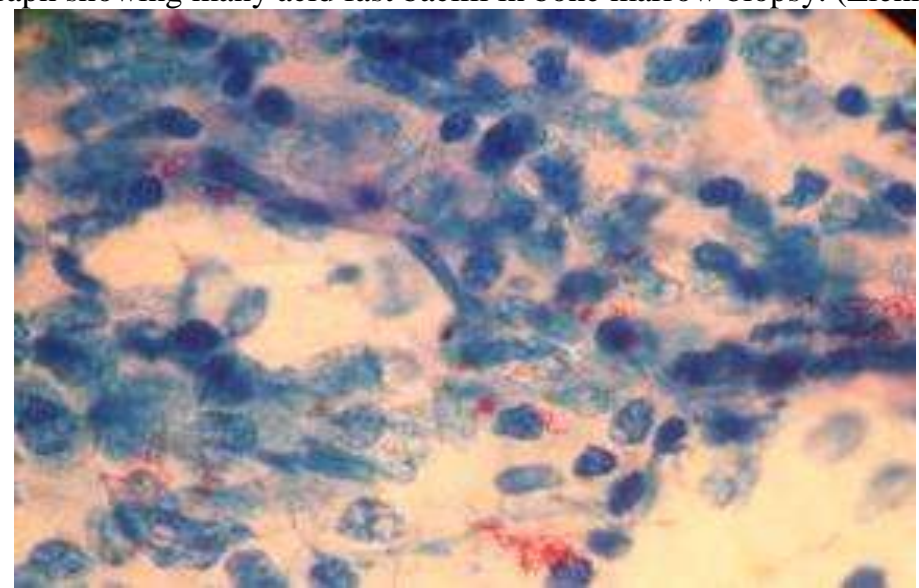

Figure 5- Photomicrograph of Bone marrow aspirate showing Acid Fast Bacilli. (Ziehl Neelson stain, x1000)

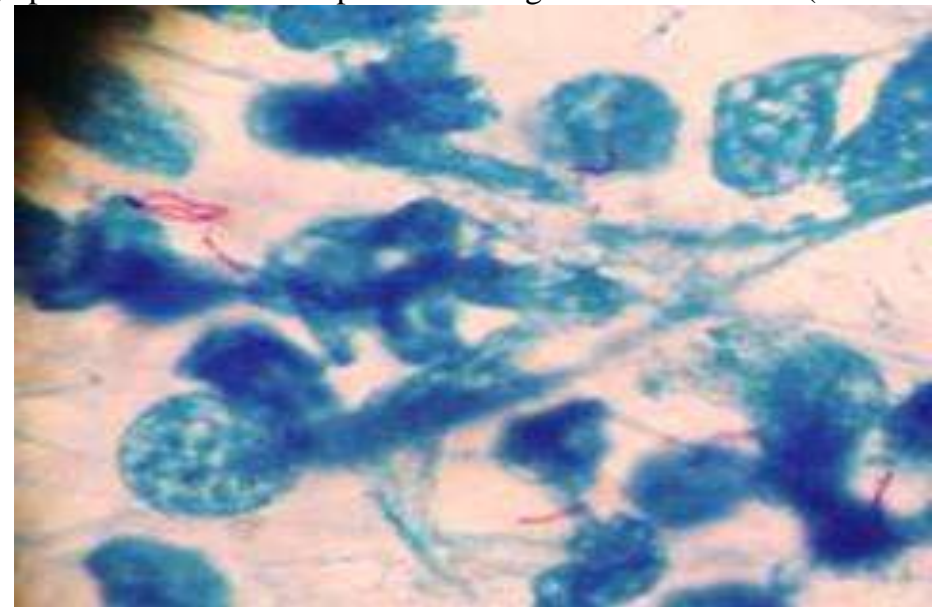

